\title{
TEMPERAMENTO DE CRIANÇAS NA ABORDAGEM DE ROTHBART: ESTUDO DE REVISÃO SISTEMÁTICA ${ }^{1}$
}

\author{
Maria Beatriz Martins Linhares ${ }^{2}$ \\ Aline Limiéri Dualibe \\ Rafaela Guilherme Monte Cassiano \\ Universidade de São Paulo, Ribeirão Preto-SP, Brasil
}

\begin{abstract}
RESUMO. O temperamento é definido pelas diferenças individuais na reatividade e autorregulação, tendo composição biológica e podendo ser influenciado pelo ambiente. O modelo psicobiológico de Mary Rothbart tem sido amplamente utilizado no estudo do temperamento. O objetivo desse estudo foi revisar sistematicamente a literatura indexada publicada nos anos de 2008 a 2011 que trata do temperamento de crianças no período do nascimento até a idade escolar, de acordo com a abordagem de Rothbart. As bases de dados utilizadas foram PubMed, PsycINFO, Scielo e Lilacs. Foram encontrados 25 artigos, os quais foram analisados por três pesquisadores quanto aos aspectos objetivo, amostra, instrumentos, resultados e conclusões. As amostras dos estudos foram predominantemente crianças norte-americanas $(48 \%)$ e europeias ( $32 \%)$, avaliadas, em sua maioria, nos primeiros três anos de idade. A maioria dos estudos (92\%) avaliou o temperamento da criança por meio de questionários de heterorrelato, sendo as mães as principais informantes. Os resultados mostraram, de um lado, a relação entre temperamento e indicadores biológicos da criança (oito estudos)m e de outro, a associação entre temperamento e características do contexto familiar (sete estudos). A relação entre temperamento e características do comportamento ou personalidade foi encontrada em quatro estudos. Os demais estudos mostraram associação entre temperamento e transtornos/síndromes, contexto familiar/escolar ou nível socioeconômico/imigração. Nos achados verificou-se que o temperamento nas fases iniciais de desenvolvimento da criança, de acordo com o modelo de Rothbart, mostrou associação com características da personalidade, problemas de comportamento e psicopatologias, podendo ser moderado por interações com o ambiente familiar.
\end{abstract}

Palavras-chave: Temperamento; crianças; desenvolvimento infantil.

\section{SEARCHING FOR THE ONTOLOGICAL BASES OF VYGOTSKY'S PSYCHOLOGY}

\begin{abstract}
Temperament is defined through the individual differences in reactivity and self-regulation, with biological composition and may be influenced by the environment. The psychobiological approach of Mary Rothbart has been broadly used in the study of temperament. The aim of the present study was to systematically review the published literature indexed between 2008 and 2011, on the topic of child temperament, from the birth period to school age, according to the Rothbart's approach. The databases used were PubMed, PsycINFO, Lilacs and Scielo. It was found 25 articles which were analyzed by three researchers as to: objective, sample, instruments, results and conclusions. Study samples were predominantly American children (48\%) and European (32\%), assessed mostly in the first three years old. Most studies (92\%) assessed the child's temperament through hetero-report questionnaires, with the mothers being the main informants. The results showed on one hand the relationship between temperament and biological indicators of child (eight studies) and, on the other hand, the association between temperament and familiar context characteristics (seven studies). The relationship between temperament and personality or behavior characteristics occurred in four studies. The rest of the studies showed an association between temperament and disorders /syndromes, family/school contexts or socioeconomic status/immigration. The finding showed that the temperament in the child early stages of development, according to the model of Rothbart, presented associations with personality characteristics, behavior problems and psychopathology, which can be moderated by the interactions with the family environment.
\end{abstract}

Key words: Temperament; children; child development..

1 Apoio e financiamento: Fundação de Amparo à Pesquisa do Estado de São Paulo (FAPESP) e Conselho Nacional de Desenvolvimento Científico e Tecnológico (CNPq).

2 Endereço para correspondência: Av. Tenente Catão Roxo, 2650, salas 52/53, Faculdade de Medicina de Ribeirão Preto, Universidade de São Paulo, Campus Universitário Monte Alegre - CEP 14.048-900, Ribeirão Preto-SP, Brasil. E-mail: linhares@fmrp.usp.br 


\title{
EL TEMPERAMENTO DE LOS NIÑOS PARA HACER FRENTE A ROTHBART: UNA REVISIÓN SISTEMÁTICA
}

\begin{abstract}
RESUMEN. Temperamento se define por las diferencias individuales en la reactividad y la auto-regulación, y la composición biológica y puede ser influenciada por el medio ambiente. Actualmente el enfoque psicobiológico de Mary Rothbart se ha utilizado ampliamente en el estudio del temperamento. El objetivo de este estudio fue revisar sistemáticamente la literatura publicada indexada en los últimos tres años, sobre el tema del temperamento del niño, desde el período de el nacimiento hasta la edad escolar, de acuerdo con el enfoque de Rothbart. Las bases de datos utilizadas fueron PubMed, PsyclNFO, LILACS y SciELO. Encontrados 25 artículos, los cuales fueron analizados por tres investigadores en cuanto a: objetivos, muestras, instrumentos, resultados y conclusiones. Muestras del estudio fueron predominantemente niños estadounidenses (48\%) y Europa (32\%) fueron evaluados, sobre todo en los tres primeros años. La mayoría de los estudios (92\%) evaluaron el temperamento del niño a través de heterorrelato y las madres eran los informantes principales. Los resultados mostraron un lado de la relación entre los estribos y los indicadores biológicos del niño (ocho estudios ) y, por otro lado, la asociación entre el temperamento y características del contexto de la familia (siete estudios). La relación entre el temperamento y características del comportamiento o de la personalidad que se encuentra en cuatro estudios. El resto de los estudios mostró una asociación entre el temperamento y los trastornos / síndromes, antecedentes familiares / educación o nivel socioeconómico / inmigración. Los resultados mostraron que el temperamento en las primeras etapas del desarrollo del niño, de acuerdo con el modelo de Rothbart, mostró asociación con las características de personalidad, problemas de conducta y psicopatología, puede ser moderado por las interacciones con el entorno familiar.
\end{abstract}

Palabras-clave: Temperamento, niños, desarrollo infantil.

O temperamento infantil pode ser estudado por meio de diversas abordagens. De acordo com o estudo de revisão de Klein e Linhares (2010), podem ser identificadas quatro principais abordagens no estudo do temperamento: a de Thomas e Chess, Bluss e Plomin, Rothbart e Kagan. Essas abordagens se diferenciam quanto à concepção e procedimentos de avaliação do temperamento; no entanto, recentes revisões da literatura mostraram que a abordagem psicobiológica de Rothbart é a mais utilizada para o estudo do temperamento infantil (Cosentino-Rocha \& Linhares, no prelo; Klein \& Linhares, 2010). Esse modelo trouxe um grande avanço ao postular que as diferenças individuais do temperamento também podem ser observadas na reatividade e no funcionamento psicofisiológico, neuroendócrino e autonômico do indivíduo (Klein \& Linhares, 2010). Com isso, essa abordagem traz avanços quanto ao estudo do temperamento ao ampliar a sua definição para um modelo psicobiológico e ao avaliar a evolução do temperamento ao longo do desenvolvimento (Shiner et al., 2012).

De acordo com Rothbart, o temperamento consiste em diferenças individuais com base constitucional na reatividade e autorregulação, nos domínios do afeto, atividade e atenção. O termo constitucional refere-se às bases relativamente biológicas do temperamento, as quais são influenciadas o tempo todo pela hereditariedade, maturação e experiência. A reatividade refere-se às características da responsividade individual a mudanças de estimulação externa ou interna. A autorregulação, por sua vez, consiste nos mecanismos usados pelo indivíduo para controlar suas reações emocionais e comportamentais a fontes de estimulação positiva ou negativa (Rothbart, 2004).

Além disso, o temperamento possui três grandes fatores, a saber, afeto negativo, extroversão e controle com esforço, os quais se diferenciam quanto à reatividade $e$ regulação das emoções básicas (Rothbart \& Bates, 2006). O afeto negativo é um dos primeiros aspectos do temperamento que surgem no desenvolvimento do indivíduo. Em seu inicio esse fator é caracterizado por formas primitivas de irritação e angústia seguidas por estados mais organizados relacionados à frustração e ao medo (Rothbart, 2004). No fator extroversão observa-se a iniciação rápida de resposta, alto nível de atividade, preferência por situações caracterizadas por estímulos de alta intensidade (exposição a riscos) e relativa inquietação em novas situações sociais (Rothbart, 2004). O controle com esforço refere-se à capacidade de focalizar a atenção, exibir satisfação em atividades de baixa intensidade e exercer o controle inibitório (Shiner et al., 2012). Este fator é, em grande parte, um produto do desenvolvimento do sistema de atenção executiva que começa no 
final do primeiro ano de vida (Rothbart, 2004). Os três fatores podem ser avaliados por meio de questionários de heterrorelato do cuidador principal e por observações realizadas em laboratório (Rothbart \& Bates, 2006).

Destaca-se que as diferenças individuais do temperamento podem, em seus extremos, constituir-se em psicopatologia ou predispor o indivíduo a esta, expondo-o a maior ou menor risco para transtornos psicopatológicos (Rothbart \& Bates, 2006). Além disso, as características do temperamento podem influenciar tanto a forma de expressão de um transtorno quanto a sua evolução e a probabilidade de sua recidiva (Rothbart, 2004).

O presente estudo visa avançar, em relação aos estudos de revisão anteriores, na compreensão sobre as metodologias utilizadas e os resultados da investigação sobre o temperamento infantil, exclusivamente na abordagem de Rothbart. Diferentemente dos anteriores, o presente estudo de revisão focalizou exclusivamente estudos sobre temperamento na abordagem de Rothbart e não teve como questão principal a relação do temperamento com o gênero como a teve o estudo de Cosentino-Rocha e Linhares (2013).

O presente estudo teve por objetivo revisar a literatura indexada, publicada no período de 2008 a 2011, sobre o tema Temperamento de crianças, considerando o período do nascimento até a idade escolar, de acordo com a abordagem psicobiológica de Rothbart. $O$ estudo visa atender a três questões principais: a) quais as idades em que 0 temperamento foi mais pesquisado; b) quais os procedimentos e instrumentos utilizados para mensurar o temperamento; c) quais os principais resultados encontrados nos estudos.

\section{MÉTODO}

A presente revisão bibliográfica foi realizada por meio da seleção de artigos empíricos nas bases de dados PubMed, PsycINFO, Lilacs e Scielo, publicados no período de janeiro de 2008 a junho de 2011. A palavra-chave utilizada na busca foi Temperament/Temperamento. No PubMed foram utilizados os seguintes limites: humans; all infant birth-23 months; newborn birth-1 month; infant 1-23 months; preschool child 2-5 years; child: 6-12 years; early childhood education; kindergarten; primary education. No Psyclnfo foi feita a combinação das seguintes palavras: (\{Early Chilhood\} OR \{Kindergarten\} OR \{Preschool Education\} OR \{Primary Education\}) AND Temperament).

Foram incluídos no estudo: artigos publicados no período de 2008 a 2011; estudos empíricos que avaliaram ou mensuraram o temperamento de crianças desde o nascimento até os doze anos de idade; artigos publicados nas línguas inglesa, portuguesa ou espanhola; e artigos somente com abordagem de Rothbart para estudo do temperamento. Foram excluídos: artigos de revisão, meta-análise, comentários, editoriais ou cartas; estudos que não tivessem como objetivo principal avaliar o temperamento de crianças na faixa do nascimento aos 12 anos de idade; artigos com outras abordagens de estudo do temperamento; e estudos com animais.

$\mathrm{Na}$ busca inicial foram encontrados 550 artigos nas bases de dados, 499 no PubMed, 50 no PsycINFO, um no Scielo e nenhum no Lilacs. Em primeiro lugar foram retirados os artigos repetidos, e em segundo lugar, após a leitura dos resumos de 452 artigos, foram aplicados os critérios de inclusão/exclusão por três pesquisadores. Foram eliminados 427 artigos, por um dos seguintes motivos: o objetivo principal do estudo não ter sido o temperamento da criança $(n=245)$; a pesquisa ter sido realizada com animais $(n=8)$; e o estudos ter utilizado amostras de faixas etárias acima dos doze anos $(n=174)$. A amostra final de estudos foi de vinte e cinco artigos.

Os artigos foram analisados de acordo com o objetivo definido no estudo, a caracterização da amostra, o delineamento adotado na pesquisa, os instrumentos de avaliação e medidas utilizados, os principais resultados, as conclusões da pesquisa e as limitações do estudo. Os estudos foram lidos e fichados de acordo com estes itens por dois pesquisadores e, posteriormente, todos os estudos foram revisados por um terceiro pesquisador.

\section{RESULTADOS}

\section{Características dos estudos}


Os vinte e cinco artigos revisados foram publicados predominantemente no ano de $2009(n=13 ; 52 \%)$, e os demais se distribuíam entre os anos de $2010(n=5 ; 20 \%), 2008(n=$ $4 ; 16 \%)$ e $2011(n=3 ; 12 \%)$. Trezes estudos foram longitudinais (52\%) (Berdan, Keane \& Calkins, 2008; Bergman, Glover Sarkar, Abbott \& O'Connor, 2010; Braungart-Rieker, HillSoderlund \& Karrass, 2010; Burney \& Leerkes, 2010; Jansen et al., 2009; Klein, Gaspardo, Martinez, Grunau \& Linhares, 2009; Pesonen et al., 2009; Roza et al., 2008; Schlotz, Jones, Godfrey \& Phillips, 2008; Volbrecht \& Goldsmith, 2010; Wasser et al., 2011; Wolff et al., 2010; Zhou et al., 2008) e os doze estudos restantes foram de corte transversal (48\%) (Berry \& Schwebel, 2009; Cook, SchoppeSullivan, Buckley \& Davis 2009; Gartstein, Pelg, Young \& Slobodskaya, 2009; John \& Mervis, 2010; Kertes et al, 2009; De Pauw, Mervielde \& Leeuwen, 2009; De Pauw, Mervielde, Leeuwen \& Clercq, 2011; Rudasill, Gallagher \& White, 2010; Scher, Hall, Zaidman-Zait \& Weinberg, 2009; Spinrad et al., 2009; Xu, Farver \& Zhang 2009; Yap et al, 2011).

As crianças que compuseram a amostra dos estudos eram procedentes predominantemente da América do Norte $(n=$ 13; sendo EUA, $n=12$ e Canadá, $n=1$ ), seguindo-se as da Europa $(n=8$; sendo Holanda, $n=3$, Bélgica, $n=2$, Inglaterra, $n=1$; Finlândia, $n=1$ e Rússia, $n=1$ ), da Ásia (China, $n=2$ ), da América do Sul (Brasil, $n=1$ ) e da Oceania (Austrália, $n=1$ ). $A$ fase de desenvolvimento de avaliação do temperamento foi realizada, na maioria dos casos, nos primeiros três anos de idade (do nascimento até um ano [28\%] e de um ano e um mês até três anos de idade [20\%]). As demais foram distribuídas entre as faixas etárias de três anos e um mês a seis anos de idade $(24 \%)$ e de seis anos e um mês a doze anos de idade (24\%). Destes estudos, apenas um avaliou crianças de onze anos de idade.

$\mathrm{Na}$ maior parte dos artigos $(n=24 ; 96 \%)$ o temperamento foi avaliado por meio de questionários de Rothbart. Em quatro artigos (16\%) houve a combinação de questionário e observação direta do comportamento em situação sistemática e estruturada de laboratório utilizando-se o Laboratory Temperament Assessment Battery, Lab-TAB, (Braungart-Rieker et al., 2010; Kertes et al.,
2009; Volbrecht \& Goldsmith, 2010; Spinrad et al., 2009). Em três artigos essas combinações foram compostas pelo Lab-TAB e o Child Behavior Questionnaire e em um artigo (Braungart-Rieker et al., 2010) pelo Lab-TAB e o Infant Behavior Questionnaire. Em apenas um estudo ocorreu somente a situação de observação em laboratório com o Lab-TAB (Bergman et al., 2010). As formas breves (short-form/very short form) do CBQ foram adotadas em cinco estudos (Cook et al., 2009; Kertes et al., 2009; De Pauw et al., 2011; Schlotz et al., 2008; Volbrecht \& Goldsmith, 2010), enquanto em um estudo foi aplicada apenas duas sub-escalas do IBQ (BraungartRicker et al., 2010).

Dos vinte e quatro estudos com relato de informantes sobre o temperamento da criança, vinte e três foram de heterorrelato. Neste e um de autorrelato. Neste último caso, os préadolescentes autoavaliavam o seu próprio temperamento. Nos vinte e quatro estudos com heterorrelato houve predomínio da mãe como principal informante, sendo que em doze estudos foi exclusivamente a mãe quem avaliou o temperamento (Berdan et al., 2008; Braungart-Ricker et al., 2010; Jansen et al., 2009; Klein et al., 2009; De Pauw et al., 2011; Roza et al., 2008; Rudasill et al., 2010; Scher et al., 2009; Schlotz et al., 2008; Spinrad et al., 2009; Wasser et al., 2011; Wolff et al., 2010). Dos demais estudos, seis tinham a mãe ou o pai como respondentes (Berry \& Schwebel, 2009; Cook et al., 2009; Gartstein et al., 2009; John \& Mervis, 2010; Kertes et al., 2009; De Pauw et al., 2009), três incluíram a mãe e o pai (Burney \& Leerkez, 2010; Pesonen et al., 2009; Volbrecht \& Goldsmith, 2010), um estudo envolveu as mães e as professoras (Xu et al., 2009), e por fim, um estudo envolveu professores, mães ou pais (Zhou et al., 2008).

\section{Principais resultados dos estudos}

A apresentação dos resultados será realizada de acordo com agrupamentos temáticos, focalizando o objetivo do estudo e as principais variáveis analisadas.

\section{Temperamento e indicadores biológicos}

O temperamento foi estudado em grupos de crianças com vulnerabilidade biológica devida a problemas de crescimento fetal, de redução de volume cerebral, de nascimento prematuro $\quad(<37$ semanas de idade 
gestacional) e síndromes. Além disso, os hormônios de cortisol, betametazona, alfamilase e testosterona, avaliados na fase pré- ou pós-natal, foram explorados na sua relação com as características de temperamento das crianças.

Os resultados mostraram que, na fase bem inicial do desenvolvimento da criança, houve variações do tamanho ventricular cerebral de fetos e neonatos avaliados na fase de 21 a 30 semanas de idade gestacional, associadas a dificuldades de temperamento em uma amostra de crianças holandesas (Roza et al., 2008). Verificou-se que as crianças que demonstraram temperamento com maior nível de atividade e maior estresse relacionados aos limites, aos seis meses de idade apresentaram menor volume ventricular cerebral nesta fase inicial do desenvolvimento. No estudo de Roza et al. (2008) não foi encontrada associação entre a circunferência da cabeça e características do temperamento das crianças avaliadas pelas mães, diferentemente do verificado nos estudos longitudinais de Pesonen et al. (2009) e Schlotz et al. (2008). Bebês finlandeses com maior peso ao nascimento e maior circunferência de cabeça (medida do crescimento fetal) na fase de dezessete semanas de gestação apresentaram temperamento com mais controle com esforço (controle inibitório, focalização da atenção, aconchego) e menor afeto negativo (timidez) em crianças aos dois anos de idade quando avaliadas pelos pais (Pesonen et al., 2009). O problema de crescimento fetal também levou a problemas de comportamento particularmente de hiperatividade - em crianças inglesas na idade de sete a nove anos. Esses problemas se mostraram exacerbados quando associados ao temperamento com menos controle com esforço avaliado pela mãe (Schlotz et al., 2008).

Os hormônios avaliados, tanto nas crianças quanto nas mães, na fase pré-natal ou pós-natal aos dois anos de idade da criança, mostraram associação com as dimensões de temperamento dessas crianças, quando avaliadas a longo prazo em diferentes fases do desenvolvimento. Bebês que foram expostos a doses repetidas de betametazona por mais de 24 horas antes do parto apresentaram mais impulsividade aos dois anos de idade do que bebês expostos por períodos iguais ou inferiores a 24 horas (Pesonen et al., 2009). Especificamente, bebês ingleses, do sexo masculino, expostos a testosterona no líquido amniótico na fase pré-natal, independentemente do nível de cortisol presente no líquido amniótico no período pré-natal, apresentaram maiores reações de medo em situação de observação na fase de catorze a dezenove meses de idade (Bergman et al., 2010).

Adicionalmente, o maior nível de cortisol basal salivar de crianças canadenses saudáveis mostrou relação com pior qualidade do sono de crianças de doze a trinta e seis meses (Scher et al., 2009). Esta pior qualidade do sono esteve também associada ao temperamento, avaliado pelas mães, com traços de mais raiva e menos controle inibitório no temperamento. Além disso, foi identificado que o nível mais alto de cortisol estava associado com temperamento com afeto negativo nas crianças avaliadas pelos professores. Por outro lado, em fase mais adiantada do desenvolvimento, verificou-se que em crianças norte-americanas na fase pré-escolar a maior reatividade alfa-amilase salivar estava relacionada a temperamentos com mais controle com esforço, denotando maior controle inibitório e regulação do comportamento, avaliados pelas mães (Spinrad et al., 2009).

Deve-se considerar, não obstante, que variáveis ambientais podem ser moderadoras da relação entre o hormônio do cortisol e o temperamento da criança, avaliados tanto por observação direta quanto por relato de pais ou mães. A qualidade da interação entre a criança e os pais mostrou ser uma variável moderadora importante para amenizar o impacto negativo da elevação dos níveis de cortisol em crianças norte-americanas extremamente sociáveis, de idade entre três e cinco anos, mas não em crianças inibidas (Kertes et al., 2009). Esses achados revelam que os pais apresentarem sensibilidade nas interações e darem suporte social à criança constituem-se em elementos de proteção para neutralizar as respostas do eixo hipotálamopituitária-adrenal (HPA). Este eixo é responsável pelas respostas do estresse no indivíduo e tem importância na depressão, portanto pode afetar o desenvolvimento das crianças. 
Uma situação estressora de dor na fase inicial do desenvolvimento foi estudada, a fim de avaliar a reatividade biocomportamental em situação de coleta de sangue durante a internação em uma Unidade de Terapia Intensiva Neonatal, em crianças brasileiras nascidas pré-termo (Klein et al., 2009). Foi examinada a associação desta reatividade com o temperamento, avaliado pelas mães, na idade entre 18 e 32 meses Foi verificado que, após o controle das variáveis idade gestacional e gravidade clínica neonatal, a maior reatividade fisiológica à dor (batimento cardíaco) durante o procedimento doloroso foi preditora de temperamento com afeto negativo, especialmente nas dimensões medo e frustração. Além disso, a maior reatividade comportamental à dor na fase neonatal foi preditora de temperamento com menor sociabilidade. Os problemas de recuperação fisiológica após o procedimento doloroso de retornar o batimento cardíaco ao nível da fase basal (antes do procedimento doloroso) associaram-se a temperamentos de mais extroversão e prazer de alta intensidade mais adiante no desenvolvimento na fase de 18 a 32 meses.

\section{Temperamento,} personalidade

\section{comportamento}

e

O temperamento foi investigado na relação com comportamentos desajustados e indicadores de psicopatologia. A relação entre temperamento, problemas de comportamento internalizantes e externalizantes e o modelo dos cinco fatores da personalidade foi estudada em uma amostra de crianças belgas pré-escolares não-clínicas de idade entre quatro e cinco anos (De Pauw et al., 2009). Primeiramente, destaca-se que o índice de emocionalidade/neuroticismo (adaptabilidade e humor, emocionalidade, afeto negativo e instabilidade emocional), baseado nas abordagens de temperamento e no modelo de personalidade, mostrou associação positiva com problemas internalizantes. Por outro lado, apenas a emocionalidade teve relação com problemas de comportamento externalizante. Quanto maior era o nível de atividade apresentada no temperamento, avaliado pelas mães ou pais, mais ocorriam problemas externalizantes, especialmente dos tipos déficit de atenção e hiperatividade. Em segundo lugar, o índice composto por consciência/controle com esforço (atividade/persistência, controle com esforço, consciência) revelou que quanto menor este índice, maior é escore de problemas externalizantes, especialmente dos problemas afetivos e transtornos invasivos do desenvolvimento.

$\mathrm{Na}$ mesma linha da integração entre temperamento e personalidade, foi analisada a relação entre esses aspectos e o risco de se machucar em crianças norte-americanas na idade de cinco a nove anos (Berry \& Schwebel, 2009). Verificou-se que o temperamento, relatado por mães, e a personalidade com alta extroversão e baixo controle com esforço aumentavam a ocorrência de ferimentos corporais ao longo do desenvolvimento. Por outro lado, o afeto negativo/neuroticismo relacionou-se com 0 grau de gravidade dos ferimentos, enquanto o afeto negativo/neuroticismo associou-se com a quantidade de ferimentos experimentados ao longo de desenvolvimento. Quando as crianças tinham baixo controle com esforço, associado com alta extroversão e baixo afeto negativo/neuroticismo, aumentava o risco de ocorrência de machucados não intencionais. Verificou-se também relação entre o temperamento avaliado pelas mães e queixas somáticas de crianças holandesas na fase de 18 meses (Wolff et al., 2010). As queixas somáticas das crianças associaram-se com temperamento caracterizado por medo e baixa reatividade/dificuldade de superar estresse, aos seis meses de idade.

Por outro lado, o temperamento pode ter efeito moderador na relação entre emoções e transtornos emocionais. Em uma amostra de crianças australianas de dez a doze anos, foi verificado que o temperamento, avaliado por meio do autorrelato, teve um efeito moderador na regulação emocional e nos sintomas depressivos (Yap et al., 2011). Os adolescentes que tinham temperamento com menos controle com esforço e alta emocionalidade negativa relataram mais sintomas depressivos. A alta emocionalidade negativa também esteve associada com comportamentos agressivos na interação de resolução de problemas com a mãe. Portanto, os pré-adolescentes com essas características de temperamento exibiam um padrão de comportamento desadaptado. 


\section{Temperamento, Transtornos e Síndromes}

Em um estudo longitudinal conduzido por De Pauw et al. (2011), o temperamento, a personalidade e o comportamento foram avaliados em uma amostra clínica de crianças belgas na fase escolar com transtornos invasivos do desenvolvimento (autismo, síndrome de Asperger e transtorno inespecífico) Neste estudo a amostra foi dividida em grupos com alto e baixo nível de sintomas e comparada com uma amostra de crianças não-clínicas (grupo controle). As crianças com transtornos apresentaram temperamento, relatado pelos pais, com mais afeto negativo e menos controle com esforço e extroversão do que o grupo-controle. As crianças com alto nível de sintomas, por sua vez, tinham mais problemas de comportamento internalizante e temperamento com mais afeto negativo, timidez e menos extroversão, afiliação e prazer de alta intensidade do que as crianças com menos sintomas. Na amostra total, foi detectado que temperamentos com alto nível de extroversão foram preditores de baixo escore de problemas internalizante e alto escore de problemas externalizante; por outro lado, temperamentos com alto nível de afeto negativo e baixo nível de controle com esforço foram preditores de alto escore de problemas tanto internalizantes quanto externalizantes.

Crianças norte-americanas com síndrome de Williams com alto nível de prejuízos sensoriais mostraram temperamento, relatado por mães ou pais, com mais raiva/frustração e tristeza e menos reatividade decrescente/capacidade de se acalmar, controle inibitório e focalização da atenção, na fase de quatro a dez anos, do que crianças com baixo nível de prejuízo sensorial (John \& Mervis, 2010).

\section{Temperamento e o contexto familiar}

O temperamento de crianças mostrou forte associação e interação com características dos cuidados parentais e práticas educativas, o que permite avançar na compreensão dos problemas de comportamento nas crianças. $\mathrm{O}$ temperamento com menos controle com esforço esteve associado com comportamento agressivo pró-ativo e reativo em crianças chinesas de cinco anos, avaliadas por mães e professores (Xu et al., 2009). Além disso, crianças com mais raiva e frustração, avaliadas por seus pais e professores, tinham mais reações agressivas identificadas pelos professores e colegas. Por outro lado, os cuidados parentais mostraram associação com características do temperamento das crianças, e filhos de pais indulgentes apresentaram temperamento com mais raiva e frustração, de acordo com a percepção tanto das mães quanto dos professores. 0 temperamento com mais busca de sensação, raiva ou frustração esteve associado com mais comportamento agressivo pró-ativo ou reativo, em crianças com baixo ou moderado controle com esforço, mas não em crianças com alto controle com esforço. Quanto mais o cuidado parental era do tipo indulgente, mais ocorria o comportamento agressivo pró-ativo nas crianças com baixo ou moderado controle com esforço e alta ou moderada busca por sensações, mas não em crianças com alto controle com esforço e moderada e baixa busca por sensações. Por outro lado, quanto mais o cuidado parental era rígido, mais ocorria agressividade pró-ativa nas crianças com alta ou moderada busca por sensações, mas não nas crianças com baixa busca por sensações.

O temperamento com traços de raiva e frustração, avaliado pelos pais e professores na fase de sete anos, e os pais serem autoritários (muito exigentes e pouco responsivos) foram preditores de comportamento externalizante aos onze anos (Zhou et al., 2008). Adicionalmente, o temperamento com menos controle com esforço e mais eventos negativos da escola (por exemplo, tirar uma nota ruim na prova ou na lição de casa, brigar ou ser mal interpretado por um amigo próximo, entre outros) levaram a mais comportamentos externalizantes, aos onze anos, em crianças educadas por pais autoritários. Em contrapartida, a presença de pais com autoridade (muito exigentes e com alta responsividade) foi preditor de menos comportamentos externalizantes.

Temperamentos com mais afeto negativo, avaliado por mães e pais, em crianças norteamericanas da faixa de quatro anos, foram preditores de baixo compartilhamento entre os pais na educação dos filhos (coparentalidade/coparenting), ou seja, críticas entre os pais sobre como educar os filhos na presença destes (Cook et al., 2009). Por outro 
lado, houve interação entre o temperamento da criança e o ajustamento conjugal dos pais. Crianças com menos afeto negativo, filhas de pais com melhor ajustamento conjugal, apresentaram uma coparentalidade com suporte entre os pais. Destaca-se que em crianças de temperamento de alto afeto negativo, o ajustamento conjugal não atuou como fator moderador da relação entre o temperamento da criança e a coparentalidade com suporte entre os pais.

Em coerência com esta visão de que a reatividade da criança pode ter impacto nas práticas educativas parentais, o estudo de Burney e Leerkes (2010) mostrou que havia maior coparentalidade, em que os parceiros dividiam tarefas de cuidados parentais, quando os bebês norte-americanos apresentavam temperamento com capacidade de se acalmar aos seis meses. Além disso, foi verificada interação entre as dimensões temperamento, avaliado por mães e pais, capacidade de se acalmar e estresse diante de uma novidade na predição da coparentalidade. Destaca-se que esses achados foram significativos apenas na percepção das mães.

Bebês norte-americanos com maior reatividade de raiva, exibida durante a situação experimental de contenção dos braços, na fase de quatro meses, apresentaram menos medo em situação estranha, observada em laboratório, na fase de oito meses (Braungart-Rieker et al., 2010). Além disso, altos níveis de reatividade ao medo foram relacionados a menor orientação ao objeto inanimado, na situação experimental de aproximação de estranhos (strange approach) e na de contenção dos braços, e associados a baixos níveis de sensibilidade materna durante a brincadeira com seu filho. Por outro lado, temperamentos, percebidos pelas mães, de mais reatividade à raiva foram relacionados a níveis mais elevados de orientação ao objeto durante a situação de aproximação de estranhos e a níveis mais baixos de sensibilidade materna durante a brincadeira. As crianças cujas mães se mostraram mais sensíveis demonstraram menos medo, dos quatro aos dezesseis meses, em comparação com as crianças cujas mães tinham menos sensibilidade. Com relação à reatividade à raiva, as crianças que tiveram maior orientação ao objeto demonstraram menos raiva dos quatro aos dezesseis meses.

O comportamento e o temperamento com controle inibitório foram avaliados em crianças gêmeas (monozigóticos e dizigóticos) norteamericanas aos três anos, para examinar o efeito preditivo da timidez e da ansiedade aos sete anos de idade no estudo de Volbrecht e Goldsmith (2010). As crianças com baixos níveis de controle inibitório no comportamento e temperamento, em associação a famílias com baixo nível de estresse, apresentaram mais timidez aos sete anos, tanto pelo relato de mães e pais quanto pela observação direta do comportamento da criança. Além disso, destaca-se que o temperamento com afeto negativo e o estresse familiar na família, associados ao comportamento inibitório da criança, na fase de três anos, foram preditores da ansiedade aos sete anos (Volbrecht \& Goldsmith, 2010).

O temperamento da criança, percebido pela mãe, mostrou associação com padrão alimentar de bebês na fase de três a dezoito meses (Wasser et al., 2011). Crianças norteamericanas de temperamento com maiores escores em riso/sorriso, nível de atividade, angústia por limitação, prazer de baixa intensidade, duração da orientação e capacidade de se acalmar mostraram duas vezes mais chance de serem alimentadas pelas mães com alimentos sólidos antes dos quatro meses de idade; portanto, crianças mais inquietas ou agitadas são alimentadas com sólidos mais precocemente do que crianças mais quietas ou calmas.

\section{Temperamento e o contexto escolar}

O temperamento de crianças norteamericanas foi examinado em sua relação com o desempenho escolar. Características do temperamento na fase de quatro anos mostraram relação com desempenho acadêmico na $3^{a}$ série escolar, sendo esta relação moderada pelo suporte emocional dos professores (Rudasill et al., 2010). O temperamento, relatado pelas mães, com traços de maior atenção e nível de atividade, avaliados aos quatro anos e meio de idade, foram associados a melhor desempenho em leitura e matemática. O suporte emocional do professor moderou a relação entre o nível de atenção da criança e o desempenho 
acadêmico, e baixo nível de atenção das crianças associado a pouco suporte emocional dos professores resultou em pior desempenho em leitura e matemática.

Paralelamente, verificou-se que os colegas também podem atuar como moderadores na relação entre temperamentos extrovertidos, avaliados pelas mães, e comportamentos externalizantes de crianças norte-americanas na fase da educação infantil (Berdan et al., 2008). Temperamentos de extroversão de crianças aos dois anos mostrou associação com comportamentos externalizantes, avaliados por seus professores um ano depois, e baixa aceitação pelos colegas de classe, que os consideram agressivos. Por outro lado, alta aceitação social atuou como um fator protetor, especificamente nas meninas altamente extrovertidas, que apresentavam maior risco de ser percebidas como agressivas pelos pares.

\section{Temperamento, nível socioeconômico e imigração}

Características disposicionais do temperamento podem estar associadas aos fatores ambientais distais do contexto do desenvolvimento da criança, como o nível socioeconômico e a cultura. O temperamento foi avaliado pelas mães na perspectiva da influência do nível socioeconômico da família em crianças holandesas de idade de cerca de seis meses (Jansen et al., 2009). O resultado mostrou associação entre nível ocupacional pouco qualificado de pais com baixa renda e de temperamento caracterizado por mais nível de atividade e medo. Diferentemente das expectativas iniciais, os pais mais escolarizados tinham crianças com mais tristeza.

Apenas um estudo focalizou a associação entre temperamento e cultura, de acordo com a percepção dos pais, analisando especialmente a aculturação das crianças russas imigrantes nos EUA e em Israel, na faixa de três a doze meses de idade (Gartstein et al., 2009). As crianças russas que viviam na cultura americana apresentaram temperamento com menos prazer de baixa intensidade e mais declínio de reatividade, em comparação com as crianças que viviam no seu país de origem. Por outro lado, foi encontrado que quando as crianças russas viviam em Israel, estas exibiam mais capacidade de se acalmar, quando comparadas aos outros dois grupos. As crianças russas que viviam em sua própria cultura demonstravam temperamento com mais extroversão do que as crianças emigrantes das outras duas culturas diferentes da sua cultura de origem.

\section{DISCUSSÃO}

A presente revisão mostra que os fatores do temperamento têm forte relação com o modelo de personalidade dos cinco fatores, revelando que, embora esses sejam diferentes constructos, o temperamento é um substrato em que se desenvolve a personalidade (Rothbart \& Bates, 2006). A personalidade tem muitos outros componentes além dos do temperamento, como a capacidade de pensar, habilidades, hábitos, valores, defesas, moral, crenças e habilidade social. Os traços da personalidade são definidos como padrões de pensamentos, emoções e comportamento, os quais apresentam uma consistência entre situações e estabilidade ao longo do tempo. Os traços do temperamento, por sua vez, apresentam consistência em diversas situações e ao longo do tempo, mas esses são limitados a processos básicos de reatividade e autorregulação e não incluem conteúdos específicos de pensamentos ou uso de defesas (Rothbart \& Bates, 2006). Além disso, o modelo psicobiológico do temperamento proposto por Rothbart mostra-se reafirmado nos estudos que demonstraram a relação entre temperamento e variáveis biológicas como, por exemplo, o hormônio do estresse cortisol (Scher et al., 2009) e a condição de nascimento pré-termo (Klein et al., 2009).

O temperamento mostrou associação com problemas de comportamento e psicopatologias. Os problemas de comportamentos externalizantes estavam associados a maior nível de atividade (De Pauw et al., 2009), menor controle com esforço (De Pauw et al., 2009; Schlotz et al., 2008; Zhou et al., 2008), maior extroversão (Berdan et al., 2008; De Pauw et al., 2011), maior raiva e frustração (Zhou et al., 2008) e maior afeto negativo associado a menor controle com esforço (De Pauw et al., 2011). Por sua vez, os problemas de comportamento 
internalizantes estavam associados a maior afeto negativo (De Pauw et al., 2009; De Pauw et al., 2011) e baixo controle com esforço (De Pauw et al., 2011). A respeito das psicopatologias, o temperamento infantil mostrou características específicas em crianças com transtornos invasivos do desenvolvimento (De Pauw et al., 2011) e com síndrome de Williams (John \& Mervis, 2010).

Destaca-se que a relação presente nos estudos entre temperamento e comportamento e temperamento e personalidade não apresenta sobreposição na avaliação desses três constructos, independentemente da idade avaliada. Isso se deve ao fato de os respectivos instrumentos de avaliação terem sido construídos e validados para medir os respectivos constructos. Ter associação entre temperamento e comportamento, assim como entre temperamento e personalidade, não significa que sejam exatamente os mesmos constructos (Rothbart \& Bates, 2006).

Nota-se que o fator controle com esforço desempenha um importante papel na organização da regulação emocional e comportamental das crianças no contexto familiar e escolar. O baixo nível de controle com esforço relacionou-se com aumento das ocorrências de ferimentos corporais (Berry \& Schwebel, 2009), sintomas depressivos (Yap et al, 2011), presença de transtornos invasivos do desenvolvimento (De Pauw et al., 2011), comportamento agressivo pró-ativo e reativo e raiva e frustração (Xu et al., 2009). A fase préescolar constitui-se em um momento de desenvolvimento de transição para a fase escolar, que exigirá comportamentos mais regulados, com controle inibitório e focalização da atenção, para enfrentar as tarefas evolutivas de produção escolar e socialização.

Destaca-se que as variáveis psicossociais próximas relativas a práticas educativas $e$ funcionamento psicológico dos pais foram moderadoras da relação entre temperamento e comportamento (Berdan et al, 2008; Volbrecht \& Goldsmith, 2010; Xu et al., 2009; Zhou et al, 2008), assim como a variável distal de qualificação profissional (Jansen, et al., 2009); portanto, temperamento é modificável pela experiência ambiental, conforme mostra Rothbart (2004). Por outro lado, o temperamento também influencia a relação da criança com o ambiente. As características do temperamento da criança podem influenciar a seleção dos nichos de desenvolvimento, expondo-a a maior ou menor risco para transtornos psicopatológicos; portanto as características do temperamento e do ambiente de cuidado podem trazer efeitos independentes ao desenvolvimento ou efeitos interativos entre variáveis, aumentando ou diminuindo o risco de transtornos de comportamento (Rothbart, 2004).

Os estudos do temperamento analisados envolveram a avaliação de crianças na fase desde o nascimento até a idade de doze anos, concentrando-se predominantemente nos primeiros três anos de idade. Apenas um estudo envolveu pré-adolescentes. Neste sentido, nota-se que esses estudos visam avaliar os traços constitucionais e disposicionais que contribuem para organização e funcionamento da personalidade em momentos iniciais da trajetória do desenvolvimento (Rothbart \& Bates, 2006).

Os países que apareceram nas publicações envolveram amostras de crianças norte-americanas e europeias, mostrando que existem poucos estudos envolvendo outros continentes, assim como raros estudos transculturais. Apenas um estudo focalizou a questão da influência cultural, que é uma variável distal do macrocontexto do desenvolvimento da criança. O fato de os instrumentos de avaliação do temperamento de Rothbart terem versões para diferentes línguas

http://www.bowdoin.edu/ sputnam/rothbarttemperament-questionnaires/) condição facilita o desenvolvimento de estudos em diferentes culturas.

$\mathrm{Na}$ avaliação do temperamento de acordo com a abordagem de Rothbart foram utilizados diversos instrumentos, que avaliaram fatores relevantes do temperamento e suas dimensões. Destaca-se que os instrumentos foram desenvolvidos para cada etapa do desenvolvimento. Os questionários mais utilizados nos estudos foram o CBQ (três a sete anos), seguido do IBQ (três a doze meses) e dos ECBQ (dezoito a trinta e seis meses), TBAQ (dezoito a vinte e quatro meses) e, por último, o EATQ (nove a quinze anos). A avaliação do temperamento por meio do procedimento de observação foi menos utilizada - apenas em quatro estudos -, utilizando o Lab-TAB (1 ano, 3-5 anos). As avaliações com o Lab-TAB consistem em 
observações realizadas em laboratório por meio de situações estruturadas realizadas com a criança a fim de eliciar determinado fator ou dimensão do temperamento. Por outro lado, os questionários avaliam todas as dimensões e fatores do temperamento por meio do heterrorelato do cuidador principal. A grande vantagem dos instrumentos elaborados por Rothbart é que os fatores podem ser encontrados nos diferentes instrumentos, o que confere a possibilidade de avaliar o temperamento na perspectiva do desenvolvimento prospectivo-longitudinal. Este tipo de delineamento foi adotado em $54 \%$ dos estudos revisados e favoreceu o estabelecimento de relações entre temperamento e desfechos comportamentais da criança.

Os questionários sobre o temperamento foram aplicados aos cuidadores principais da criança, o que significa que o objeto da avaliação é a percepção do outro sobre a criança. De forma semelhante à de estudos de revisão anteriores (Cosentino-Rocha \& Linhares, no prelo; Klein \& Linhares, 2010), predominaram os relatos das mães sobre seus filhos em comparação com os dos pais e professores. A mãe informante tem a vantagem de acompanhar o desenvolvimento da criança mais de perto e poder descrevê-lo em diferentes contextos. Ela é testemunha do desenvolvimento e consegue precisamente descrever as características comportamentais exibidas pelas crianças.

Não obstante, deve-se ter o cuidado de assegurar a integridade psicológica da mãe, tanto do ponto de vista afetivo quanto do cognitivo, para que não haja vieses nas informações obtidas e se possa assegurar maior confiabilidade nas informações prestadas. Os múltiplos informantes sobre o temperamento das crianças têm a vantagem de corrigir possíveis vieses na percepção do temperamento da criança. Em três estudos houve relatos tanto da mãe quanto do pai sobre o temperamento das crianças, enquanto apenas um estudo (Cook et al., 2009) calculou a correlação entre as respostas dos pais, as quais se mostraram significativamente correlacionadas com a avaliação do afeto negativo, único fator do temperamento avaliado nesse estudo. Os outros dois estudos (Burney \& Leekers, 2010; Volbrecht \& Goldsmith, 2010) calcularam apenas a consistência interna dos dois relatos em separado. Além disso, o estudo de Burney e Leerkes (2010) apresentou diferença na avaliação do temperamento feita pelos pais nas dimensões estresse diante de limites e capacidade de se acalmar. Os autores atribuem essa diferença ao fato de as mães do estudo passarem mais tempo com seus filhos do que os pais.

As combinações entre avaliações de pais e professores foram pouco encontradas na presente revisão (Xu et al., 2009; Zhou et al., 2008). A avaliação do temperamento nos ambientes familiar e escolar permite avaliar melhor a estabilidade dos traços disposicionais do temperamento da criança em diferentes contextos.

O autorrelato também foi pouco identificado nas avaliações do temperamento. Aliás, o autorrelato de fato é mais adequado a partir da fase escolar e pré-adolescência. Na presente revisão verificou-se que em apenas um estudo os pré-adolescentes avaliaram o seu próprio temperamento com menor controle com esforço, o que foi associado a mais sintomas depressivos (Yap et al., 2011).

A presente revisão da literatura mostra algumas lacunas no tocante à relação entre os indicadores de temperamento da criança e os dos seus cuidadores principais. Além disso, verifica-se grande concentração em amostras de crianças norte-americanas, o que revela a necessidade de estudos transculturais sobre o temperamento de crianças. Verificou-se que nos estudos havia pouca informação sobre as características relativas à integridade psicológica dos que informaram a respeito do temperamento das crianças. Estudos futuros precisarão incluir nos critérios de exclusão indicadores emocionais e de limitação cognitiva que possam interferir na percepção dos informantes sobre a criança. Além disso, é importante fazer novos estudos sobre o temperamento na perspectiva longitudinal das pessoas. Estes estudos devem começar quando estas ainda são crianças e ir até sua fase adulta, considerando a perspectiva desenvolvimental.

\section{REFERÊNCIAS}

Berdan, L. E., Keane, S. P., \& Calkins, S. D. (2008). Temperament and externalizing behavior: social preferences and perceived acceptance as protective factors. Developmental Psychology, 44(4), 957-968.

Bergman, K., Glover, V., Sarkar, P., Abbott, D. H., \& O'Connor, T. G. (2010). In utero cortisol and 
testosterone exposure and fear reactivity in infancy. Hormones and Behavior, 57(3), 306-312.

Berry, J. W., \& Schwebel, D. C. (2009). Configural approaches to temperament assessment: implications for predicting risk of unintentional injury in children. Journal of Personality, 77(5), 1381-409.

Braungart-Rieker, J. M., Hill-Soderlund, A. L., \& Karrass, J. (2010). Fear and anger reactivity trajectories from 4 to 16 months: The roles of temperament, regulation, and maternal sensitivity. Developmental Psychology, 46(4), 791-804.

Burney, R. V., \& Leerkes, E. M. (2010). Links between mothers' and fathers' perceptions of infant temperament and coparenting. Infant Behavior Development, 33(2), 125-135.

Cook, J. C., Schoppe-Sullivan, S. J., Buckley, C. K., \& Davis, E. F. (2009). Are some children harder to coparent than others? Children's negative emotionality and coparenting relationship quality. Journal of Family Psychology, 23(4), 606-610.

Cosentino-Rocha, L., \& Linhares, M. B. M. (2013). Temperamento e gênero. Paidéia, 23(4), 606610.

De Pauw, S. S. W., Mervielde, I., \& Leeuwen, K. G. V. (2009). How are traits related to problem behavior in preschoolers? Similarities and contrasts between temperament and personality. The Journal of Abnormal Child Psychology, 37(3), 309-325.

De Pauw, S. S., Mervielde, I., Van Leeuwen, K. G., \& De Clercq, B. J. (2011). How temperament and personality contribute to the maladjustment of children with autism. Journal of Autism Developmental Disorders, 41(2), 196-212.

Gartstein, M. A., Peleg, Y., Young B. N., \& Slobodskaya, H. R. (2009). Infant temperament in Russia, United States of America, and Israel: Differences and similarities between RussianSpeaking families. Child Psychiatry and Human Development, 40(2), 241-256.

Jansen, P. W., Raat, H., Mackenbach, J. P., Jaddoe, V. W. V., Hofman, A., Verhulst, F. C., \& Tiemeier, H. (2009). Socioeconomic inequalities in infant temperament. Social Psychiatry and Psychiatric Epidemiology, 44(2), 87-95.

John, A. E., \& Mervis, C. B. (2010). Sensory modulation impairments in children with Williams Syndrome. American Journal of Medical Genetics Part C Seminars in medical genetics, 154C(2), 266-276.

Kertes, D. A., Donzella, B., Talge, N. M., Garvin, M. C., Ryzin, M. J. V., \& Gunnar, M. R. (2009). Inhibited temperament and parent emotional availability differentially predict young children's cortisol responses to novel social and nonsocial events. Developmental Psychobiology, 51(7), 521-32.

Klein, V. C., \& Linhares, M. B. M. (2010). Temperamento e desenvolvimento: revisão sistemática da literatura. Psicologia em Estudo, 15(4), 821-829.

Klein, V. C., Gaspardo, C. M., Martinez, F. E., Grunau, R. E., \& Linhares, M. B. M. (2009). Pain and distress reactivity and recovery as early predictors of temperament in toddlers born preterm. Early Human Development, 85(9), 569576.

Pesonen, A., Räikkönen, K., Lano, A., Peltoniemi, O., Hallman, \& M., Kari, M. A. (2009). Antenatal betamethasone and fetal growth in prematurely born children: Implications for temperament traits at the age of 2 years. Pediatrics, 123(1), e31-7.

Rothbart, M. K. (2004). Commentary: Differentiated measures of temperament and multiple pathways to childhood disorders. Journal of Clinical Child and Adolescence Psychology,33(1), 82-87.

Rothbart, M. K., \& Bates, J. E. (2006).Temperament. In W. Damon, R. M. Lerner \& N. Eisenberg (Eds.), Handbook of child psychology: social, emotional and personality development (Vol. 3, 6th ed, pp. 99-165), New York: John Wiley and Sons.

Roza, S. J., Govaert, P. P., Lequin, M. H., Jaddoe, V. W. V., Moll H. A., Steegers E. A. P., Hofman, A., Verhulst, F. C., \& Tiemeier, H. (2008). Cerebral ventricular volume and temperamental difficulties in infancy: The Generation R Study. Journal of Psichyatry and Neuroscience, 33 (5), 431-9.

Rudasill, K. M., Gallagher, K. C., \& White, J. M. (2010). Temperamental attention and activity, classroom emotional support, and academic achievement in third grade. Journal of School Psychology, 48(2), 113-134.

Scher, A., Hall, W. A., Zaidman-Zait, A., \& Weinberg, J. (2009). Sleep Quality, Cortisol Levels and Behavioral Regulation in Toddlers. Developmental Psychobiology, 52(1), 44-5.

Schlotz, W., Jones, A., Godfrey, K. M., \& Phillips, D. I. W. (2008). Effortful control mediates associations of fetal growth with hyperactivity and behavioral problems in 7-to-9-years-old children. Journal of Child Psychology and Psychiatry, 49(11), 1228-1236.

Shiner, R. L., Buss, K. A., McClowry, S. G., Putnam, S. P., Saudino, K. J., \& Zentner, M. (2012). What Is Temperament Now? Assessing Progress in Temperament Research on the Twenty-Fifth Anniversary of Goldsmith et al. (1987). Child Development Perspectives, 6(4), 436-444.

Spinrad, T. L., Eisenberg, N., Granger, D. A., Eggum, N. D., Sallquist, J., Haugen, R. G., Kupfer, A., \& Hofer, C. (2009). Individual differences in preschoolers' salivary cortisol and alpha-amylase reactivity: Relations to temperament and maladjustment. Hormones and Behavior, 56(1), 133-139.

Volbrecht, M. M., \& Goldsmith, H. H. (2010). Early temperamental and family predictors of shyness and anxiety. Developmental Psychology, 46(5), 1192-1205. 
Wasser, H., Bentley, M., Borja, J., Goldman, B. D., Thompson, A., Slining, M., \& Adair, L. (2011). Infants perceived as "fussy" are more likely to receive complementary food before 4 months. Pediatrics, 127(2), 229-237.

Wolff, N., Darlington, A., Hunfeld, J., Verhulst, F., Jaddoe, V., Hofman, A., Passchier, J., \& Tiemeier, H. (2010). Determinants of somatic complaints in 18-month-old children: The Generation R Study. Journal of Pediatric Psychology, 35 (3), 306-316.

Xu, Y., Farver, J. A. M., \& Zhang, Z. (2009). Temperament, Harsh and Indulgent Parenting, and Chinese Children's Proactive and Reactive Aggression. Child Development, 80(1), 244-258.

Yap, M.B.H., Allen. N. B., O'Shea M., Parsia, P., Simmons, J.G., \& Sheeber, L. (2011). Early adolescents' temperament, emotion regulation during mother-child interactions, and depressive symptomatology. Development and Psychopathology, 23(1), 267-282.
Zhou, Q., Wang, Y., Deng, X., Eisenberg, N., Wolchik, S. A., \& Tein, J. (2008). Relations of parenting and temperament to chinese children's experience of negative life events, coping efficacy, and externalizing problems. Child Development, 79(3), 493-513.

Recebido em 09/04/2013 Aceito em 09/10/2013

Maria Beatriz Martins Linhares: professora associada do Departamento de Neurociências e Ciências do Comportamento da Faculdade de Medicina de Ribeirão Preto (USP).

Aline Limiéri Dualibe: Universidade de São Paulo, Ribeirão Preto-SP, Brasil.

Rafaela Guilherme Monte Cassiano: mestre pela Faculdade de Filosofia Ciências e Letras de Ribeirão Preto (USP) e doutoranda pela Faculdade de Medicina de Ribeirão Preto (USP). 